\section{Relación miofuncional y tratamiento de ortodoncia en paciente con síndrome de Turner. Reporte de caso}

\section{Myofunctional relation and orthodontic treatment in patient with Turner syndrome. Case report}

\section{Resumen}

El síndrome de Turner es un desorden cromosómico, generado por la pérdida parcial o total de un cromosoma $\mathrm{X}$. Las mujeres que lo presentan, pueden tener una serie de alteraciones físicas y funcionales. El objetivo de este estudio es exponer el caso de una paciente con diagnóstico de síndrome de Turner, hipotonía muscular y maloclusión severa que fue tratada con terapia muscular craneofacial, combinada con ortodoncia en la Unidad de Malformación Craneofacial de la Facultad de Odontología de la Universidad de Chile.

Palabras clave: Hipotonía; Ortodoncia; Síndrome de Turner.

\section{Caso Clínico}

\author{
Noemi Leiva ${ }^{1, a, b}$, Vanessa Fuentes ${ }^{1, a}$, \\ Francisca Ayala ${ }^{1, a}$, Carolina Stange ${ }^{1, a}$ \\ ${ }^{1}$ Universidad de Chile. Facultad de Odontología. \\ Unidad de Malformaciones Craneofaciales. \\ Santiago, Chile. \\ a Cirujana Dentista. \\ ${ }^{\mathrm{b}}$ Especialista en Ortodoncia y Ortopedia \\ Maxilofacial. \\ Correspondencia: \\ Vanessa Fuentes \\ Correo electrónico: vane.fuentes.f@gmail.com \\ General Holley 2381, oficina 606 torre B, \\ Providencia, Santiago, Chile. \\ Coautoras: \\ Noemi Leiva \\ leivanoemi@yahoo.com \\ Francisca Ayala \\ francisca@ayalajimenez.com \\ Carolina Stange \\ carostange@hotmail.com
}

Conflicto de intereses: Sin conflictos de intereses. Fuente de financiamiento: Autofinanciado.

Fecha de recepción: 12/09/18

Fecha de aceptación: 03/11/18

\begin{abstract}
The Turner Syndrome is a chromosome disorder produced by the total or partial absent of an X chromosome. The women who presents Turner syndrome can have a series of physical and functional alterations. The aim of this study is to present the case of a patient diagnosed with Turner syndrome, muscular hypotony and severe malocclusio treated with craneofacial muscular therapy combined with orthodontics at the Craneofacial Malformations Unit of the Faculty of Dentistry, University of Chile.
\end{abstract}

Keywords: Hypotony, muscle; Orthodontics; Turner syndrome. 


\section{Introducción}

El síndrome de Turner es un desorden genético, provocado por la pérdida parcial o total del segundo cromosoma X. Dentro de las características generales de las mujeres que padecen síndrome de Turner, se encuentra la disgenesia gonadal, estatura baja, alteraciones oftalmológicas, auditivas, cardíacas, renales y óseas ${ }^{1,2}$.

En el territorio craneofacial, se incluye una forma de cara triangular, nariz corta, mentón pequeño, orejas de inserción baja, paladar alto y alteración oclusal.

En estudios cefalométricos realizados, se ha descrito que el maxilar y la mandíbula presentan una posición retrognática, con una rotación posterior, siendo el largo de la base craneal posterior de menor tamaño ${ }^{3,4}$.

Además, se ha encontrado, que las mujeres con síndrome de Turner tienen un pobre desempeño motor general, debido a una hipotonía muscular y una fuerza muscular reducida, lo que puede generar grandes impedimentos en su motricidad fina y gruesa en el desarrollo de su vida diaria ${ }^{2,5-7}$.

En este reporte, se presenta el caso de una paciente, sexo femenino que al ingreso presentaba 7 ańos de edad, diagnosticada en la infancia con síndrome de Turner, y presencia de una alteración del tono muscular, que afectaba principalmente musculatura facial y postural, junto con el desarrollo de una maloclusión severa, con vulnerabilidad socio económica. El objetivo de este trabajo es presentar el tratamiento que se realizó para corregir la hipofunción de la musculatura, oclusión y alineamiento en dientes, para así conseguir estabilidad funcional y estética.

\section{Reporte del caso}

Paciente sexo femenino, 7 años, diagnosticada con una hipotonía muscular generalizada, asociada a una malfor- mación oclusal severa, es ingresada a la Unidad de Malformación Craneofacial de la Facultad de Odontología de la Universidad de Chile, para evaluación, ya que esta condición alteraba oclusión, labios, estética y posición de la lengua.

A los 6 meses de vida, se le realizó cariograma, que informó en el resultado un cariotipo 46, XX, y un diagnóstico de síndrome de Turner.

Al examen fonoaudiológico presentaba serias alteraciones, ingresó a tratamiento por presencia de nódulos en cuerdas vocales. El estado físico general indicaba problemas posturales y de equilibrio, una marcha asincrónica, pie cavo y mano derecha con cuarto dedo ausente.

Sin antecedentes familiares de fisuras orales, ni anomalías craneofaciales, presentaba como antecedente quirúrgico, amigdalectomía a los 6 años de edad.

Hallazgos clínicos. Se realizó examen extraoral, donde se observó un perfil convexo, musculatura facial no expresiva, comportamiento deglutorio alterado, incompetencia labial, patrón respiratorio bucal y voz con tono bajo para su edad y sexo.

Al examen clínico intraoral se observó dentición mixta de primera fase, paladar ojival, incisivos superiores protruidos y severo apińamiento, con falta de espacio. En el arco inferior se encontró una lengua hipotónica, cinco incisivos, falta de espacio, discrepancia negativa y caries.

En la relación oclusal, en sentido vertical presentaba una mordida abierta anterior, en sentido transversal mordida cruzada derecha y en sentido sagital resalte aumentado. En la Figura 1 se observa examen intraoral y facial, en la Figura 2, se observa examen de pies y manos.

Evaluación diagnóstica. Se realizó estudio radiográfico con ortopantomografía, donde se observó piezas 1.3,
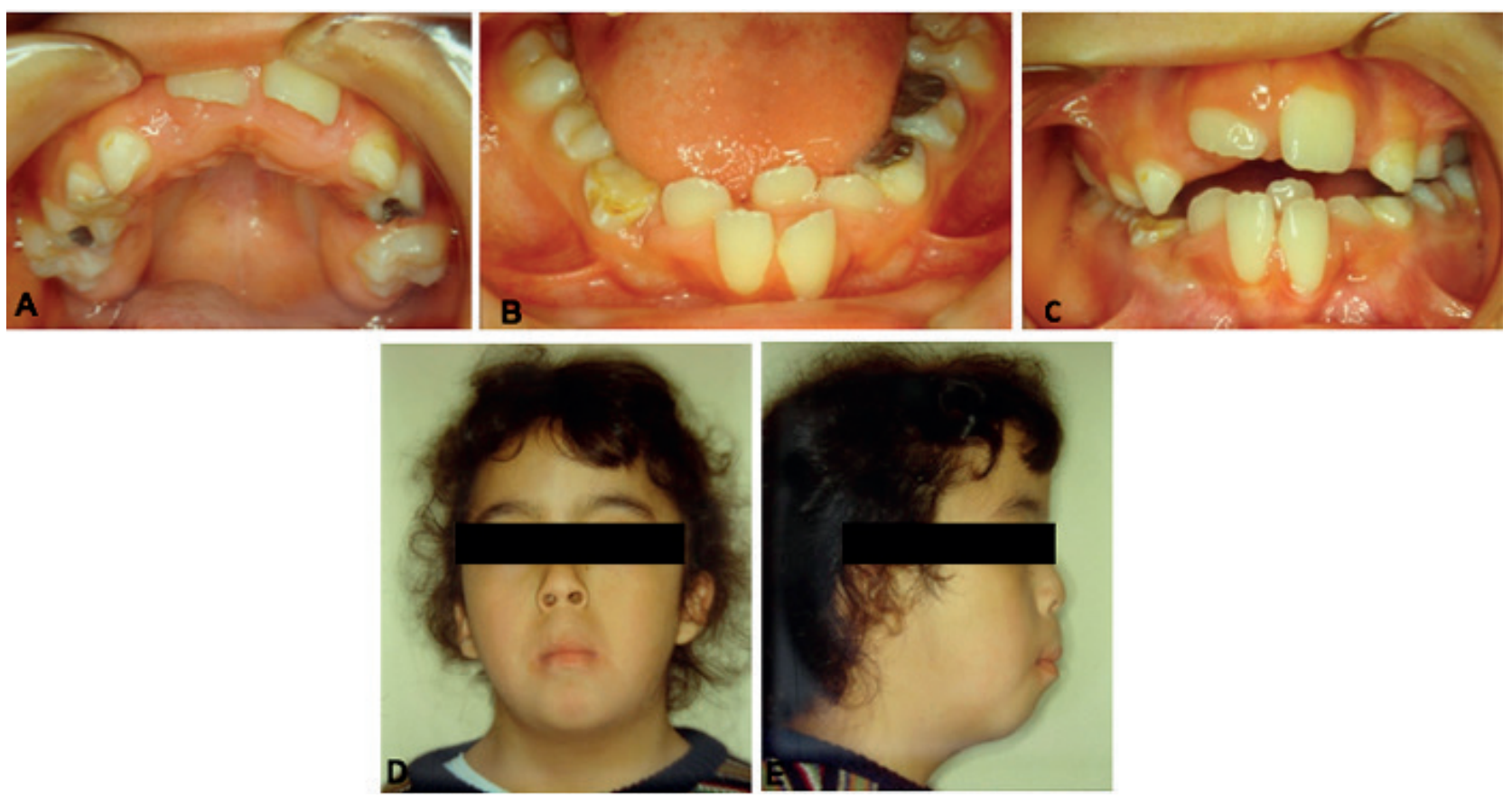

Figura 1. A. Arcada superior, incisivos en erupción. B. Arcada inferior, se observan cinco incisivos inferiores en erupción. C. Oclusión de frente. D. Cara de frente, cierre labial forzado. E. Perfil convexo 
$1.4,1.5,1.7,2.4,2.5,2.7,3.3,3.5,3.7,4.4,4.7$ en evolución intraósea y agenesia de pieza 4.5. Se solicitó además una telerradiografía lateral de perfil, que indicó la presencia de clase II esqueletal, tipo dolicofacial, retrognatia maxilar y mandibular, incisivos superiores e inferiores con una protrusión moderada y labio inferior con protrusión severa (Figura 3). Los análisis cefalométricos son presentados en las Tablas 1-4. Además, se solicitó un RhinoScan, donde se confirmó el patrón de respiración bucal.

El objetivo del tratamiento fue lograr un sello correcto durante la deglución y cierre labial, así como una adecuada actividad muscular, la corrección de mordida cruzada y línea media, alineación de ambas arcadas y la nivelación de las piezas dentarias.

Intervenciones terapéuticas. Se realizó una primera etapa de tratamiento, que consistió en la estabilización muscular; comenzó con terapia física, realizada por kinesiólogo, con ejercicios de equilibrio postural además de control respiratorio. Luego, ejercicios miofuncionales de estimulación de los músculos orbiculares de los labios y expresión de la sonrisa, además de apertura y cierre labial, después se incorporaron ejes de deglución, donde la dificultad estuvo en lograr un sellado palatino. Los ejercicios se controlaban de forma mensual hasta lograr el objetivo de adosamiento lingual, sellado labial, mejor movilidad facial y tono muscular. Esta fase tuvo una duración de 3 años, que involucró el aprendizaje y refuerzo de los ejercicios. La cooperación del paciente fue de vital importancia para la evolución positiva del tratamiento ya que se destinaban refuerzos para realizarlos en casa. En la Figura 4 se observa paciente luego de meses de tratamiento kinesiológico.

En una segunda etapa, con el fin de corregir la mordida cruzada derecha, se instaló una barra palatina, activándose mensualmente. Además, se cementó una barra lingual para lograr anclaje posterior en la arcada inferior. Esta fase tuvo una duración de 6 meses.

Se decidió comenzar la etapa ortodóntica a los 10 años, se instaló multi brackets en ambas arcadas con arcos de Nitinol 0,014", manteniendo los aparatos de expansión. Se realizaron exodoncias de las piezas 3.1 supernumeraria, 1.4, 2.4 y 3.4, debido a la falta de espacio (discrepancia negativa). Para distalizar piezas superiores e inferiores se utilizó fuerza elástica, Alastic en distintos segmentos de forma secuencial y retroligaduras. Para de-
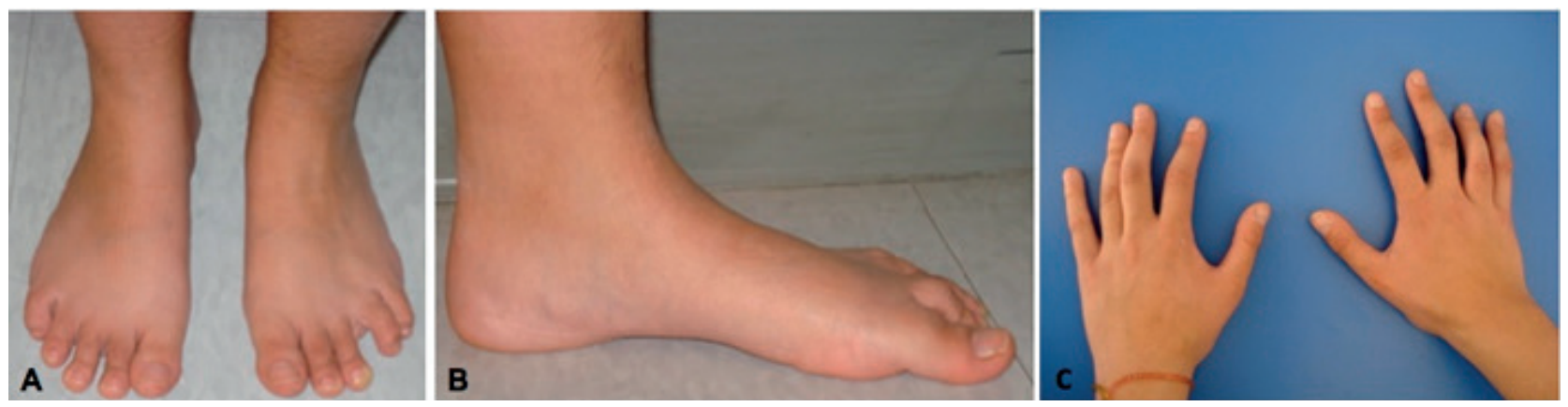

Figura 2. A. y B. Pies cavos. C. Mano derecha con cuarto dedo ausente y doble quinto dedo
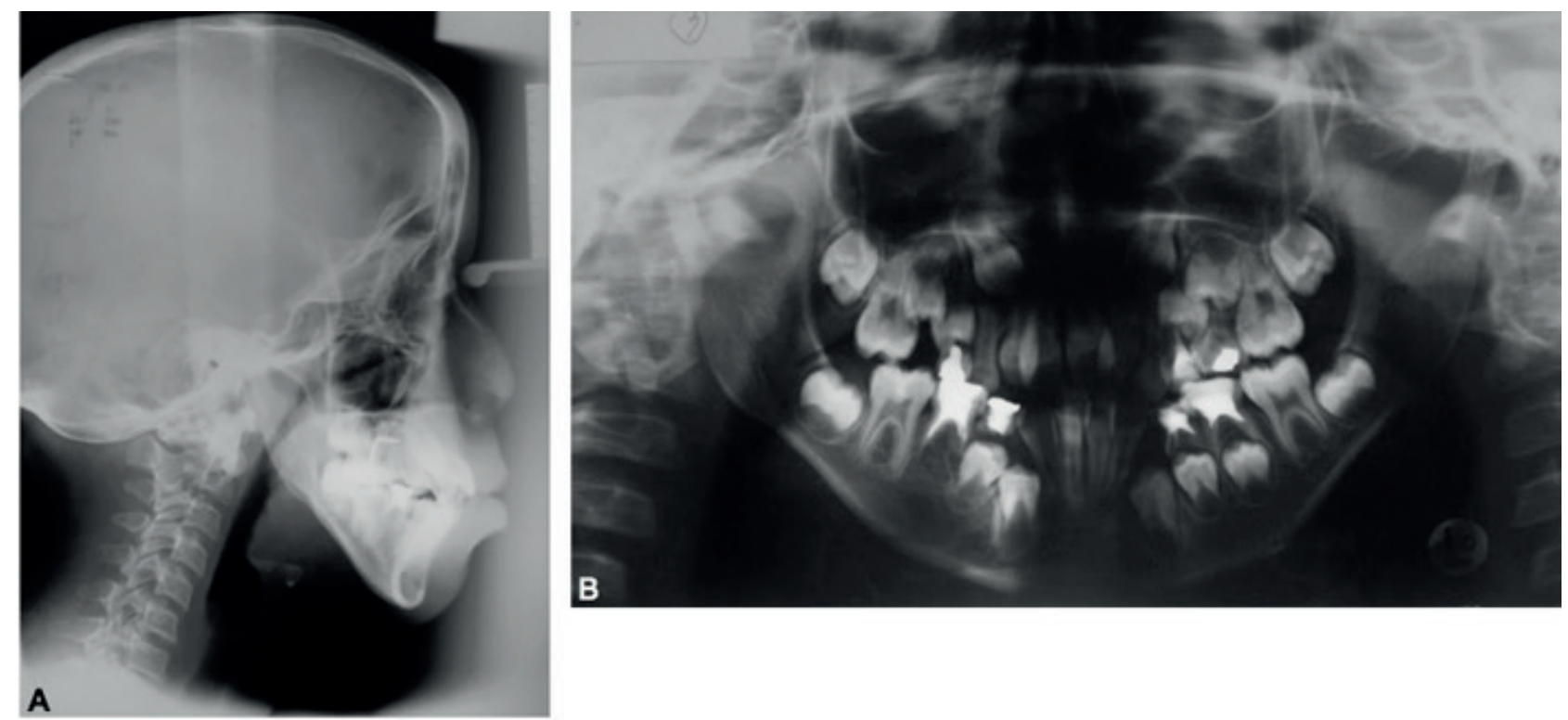

Figura 3. A. Telerradiografía lateral de perfil inicial. B. Ortopantomografía inicial 

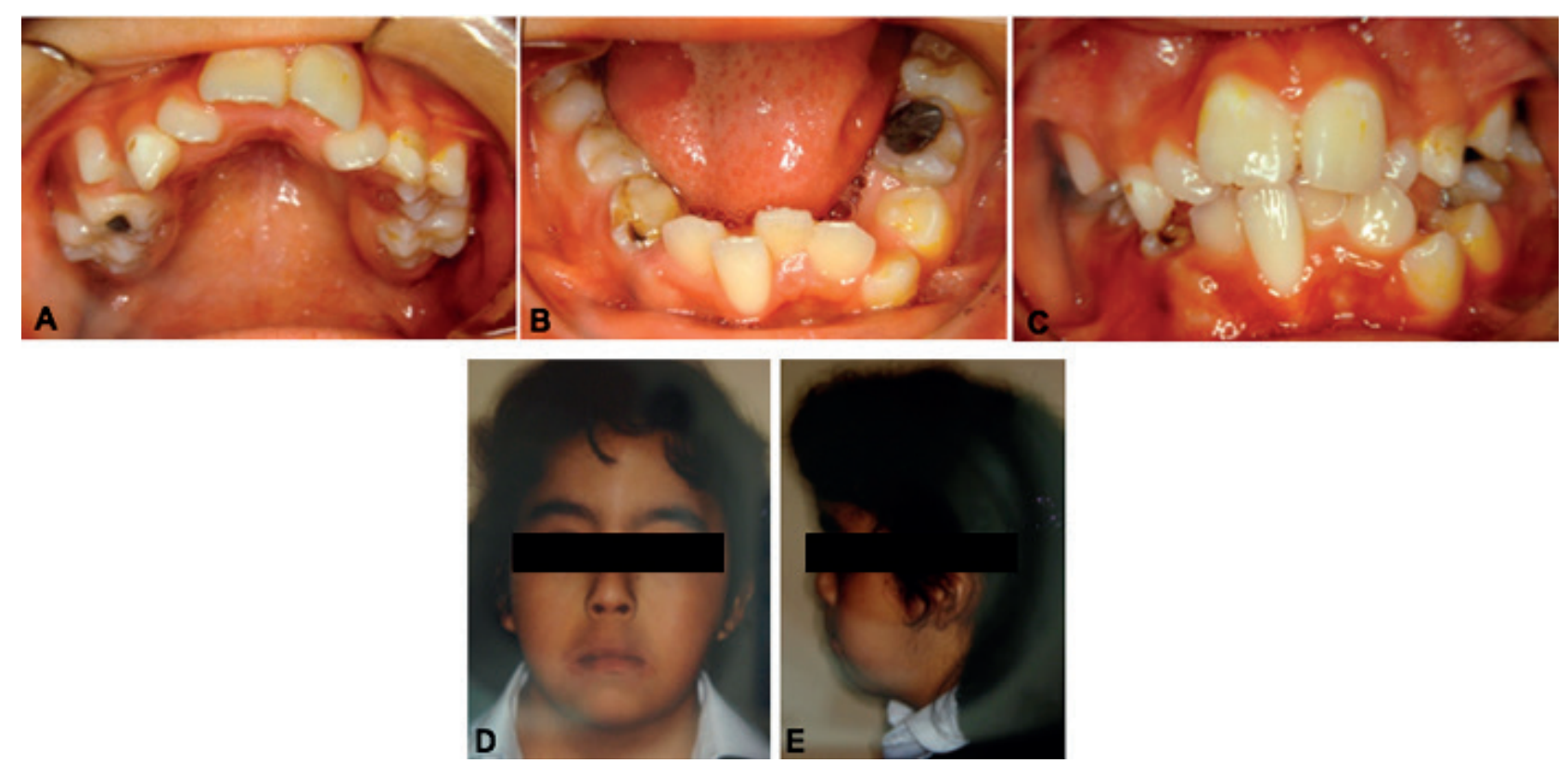

Figura 4. A. y B. Arcadas superior e inferior. C. Oclusión de frente. D. Fotografía de frente, mejor cierre labial. E. Fotografía de perfil

Tabla 1. Análisis cefalométrico de Steiner

\begin{tabular}{lccc}
\hline Variable & Resultado & Norma & Desviación clínica \\
\hline Ángulo SNA & $70,7^{\circ}$ & $82,0^{\circ}$ & $-3,8$ \\
Ángulo SNB & $76,0^{\circ}$ & $80,0^{\circ}$ & $-4,3$ \\
Mx1- NA & $3,7 \mathrm{~mm}$ & $2,0 \mathrm{~mm}$ & $0,8 \mathrm{~mm}$ \\
Ángulo Mx1- NA & $6,0^{\circ}$ & $4,0^{\circ}$ & 0,7 \\
Md1-NB & $7,8 \mathrm{~mm}$ & $4,0 \mathrm{~mm}$ & $1,3 \mathrm{~mm}$ \\
Ángulo Md1- NB & $29,4^{\circ}$ & $25,9^{\circ}$ & 0,7 \\
Po-NB & $-2,5 \mathrm{~mm}$ & $1,0 \mathrm{~mm}$ & $-2,0 \mathrm{~mm}$ \\
Plano Oclusal-SN & $32,0^{\circ}$ & $14,0^{\circ}$ & 5,2 \\
Ángulo Go- Gn- SN & $66,0^{\circ}$ & $32,0^{\circ}$ & 7,5 \\
FMA & $52,4^{\circ}$ & $25,0^{\circ}$ & $4,6^{\circ}$ \\
FMIA & $51,2^{\circ}$ & $65,0^{\circ}$ & $-2,3^{\circ}$ \\
IMPA & $76,4^{\circ}$ & $90,0^{\circ}$ & $-2,3^{\circ}$ \\
\hline
\end{tabular}

Tabla 2. Análisis cefalométrico de Jarabak

\begin{tabular}{lccc}
\hline Variable & Resultado & Norma & Desviación clínica \\
\hline Ángulo de la silla & $138,7^{\circ}$ & $123,0^{\circ}$ & 3,1 \\
Ángulo articular & $135,6^{\circ}$ & $143,0^{\circ}$ & $-1,2$ \\
Ángulo Goniaco & $151,6^{\circ}$ & $130,0^{\circ}$ & 3,1 \\
Ángulo Go- GN-SN & $66,0^{\circ}$ & $32,0^{\circ}$ & 7,5 \\
Base craneal anterior & $65,7 \mathrm{~mm}$ & $68,4 \mathrm{~mm}$ & $-0,9 \mathrm{~mm}$ \\
Base craneal posterior & $23,2 \mathrm{~mm}$ & $32,0 \mathrm{~mm}$ & $-2,9 \mathrm{~mm}$ \\
Ángulo goniaco superior & $58,5^{\circ}$ & $53,5^{\circ}$ & $1,7^{\circ}$ \\
Ángulo gonion inferior & $93,1^{\circ}$ & $72,5^{\circ}$ & 6,9 \\
Altura de la rama & $28,4 \mathrm{~mm}$ & $44,0 \mathrm{~mm}$ & $-3,1 \mathrm{~mm}$ \\
SNA & $70,7^{\circ}$ & $82,0^{\circ}$ & $-3,8$ \\
SNB & $67,0^{\circ}$ & $80,0^{\circ}$ & $-4,3^{\circ}$ \\
ANB & $3,7^{\circ}$ & $2,0^{\circ}$ & $0,8^{\circ}$ \\
Altura facial posterior & $47,8 \mathrm{~mm}$ & $74,3 \mathrm{~mm}$ & $-5,1 \mathrm{~mm}$ \\
Altura facial anterior & $122,4 \mathrm{~mm}$ & $119,0 \mathrm{~mm}$ & $0,5 \mathrm{~mm}$ \\
Altura facial post/ant. & $39,1^{\circ}$ & $62,0^{\circ}$ & $-5,7 \%$ \\
Ángulo interincisivo & $117,7^{\circ}$ & $130,0^{\circ}$ & $-2,1$ \\
Convexidad & $5,4 \mathrm{~mm}$ & $1,7 \mathrm{~mm}$ & $1,9 \mathrm{~mm}$ \\
ANS- Mentón & $71,2 \mathrm{~mm}$ & $58,9 \mathrm{~mm}$ & $2,0 \mathrm{~mm}$ \\
Nasion- Mentón & $122,4 \mathrm{~mm}$ & $105,6 \mathrm{~mm}$ & $2,8 \mathrm{~mm}$ \\
ANS-Me / Nasion - Me & $58,1^{\circ}$ & $55,0^{\circ}$ & $1,0^{\circ}$ \\
\hline
\end{tabular}


Tabla 3. Análisis cefalométrico de Ricketts

\begin{tabular}{lccc}
\hline Variable & Resultado & Norma & Desviación clínica \\
\hline Relaciones craneales & & & \\
Base craneal anterior & $56,5 \mathrm{~mm}$ & $56,2 \mathrm{~mm}$ & $0,1 \mathrm{~mm}$ \\
Base craneal posterior & $42,0 \mathrm{~mm}$ & $57,3 \mathrm{~mm}$ & $-4,6 \mathrm{~mm}$ \\
Deflección craneal & $25,9^{\circ}$ & $27,3^{\circ}$ & $-0,5$ \\
Posición del Porión & $-36,5 \mathrm{~mm}$ & $-39,3 \mathrm{~mm}$ & $1,3 \mathrm{~mm}$ \\
Posición de la rama & $69,7^{\circ}$ & $76,0^{\circ}$ & $-2,1^{\circ}$ \\
Profundidad del maxilar & $84,3^{\circ}$ & $90,0^{\circ}$ & $-1,9$ \\
Altura del maxilar & $68,3^{\circ}$ & $53,6^{\circ}$ & $4,9^{\circ}$ \\
SN- Plano palatino & $17,6^{\circ}$ & $7,3^{\circ}$ & $2,9^{\circ}$ \\
Profundidad facial & $79,3^{\circ}$ & $87,0^{\circ}$ & $-2,6$ \\
Eje facial & $79,0^{\circ}$ & $90,0^{\circ}$ & $-3,1^{\circ}$ \\
Plano mandibular & $52,4^{\circ}$ & $25,5^{\circ}$ & 6,0 \\
Altura facial total & $78,8^{\circ}$ & $60,0^{\circ}$ & 6,3 \\
Cono facial & $48,3^{\circ}$ & $68,0^{\circ}$ & $-5,6^{\circ}$ \\
Relaciones MX/MD & & & \\
Convexidad del maxilar & $5,4 \mathrm{~mm}$ & $1,7 \mathrm{~mm}$ & $1,9 \mathrm{~mm}$ \\
Altura del cuerpo mand. & $66,4 \mathrm{~mm}$ & $67,4 \mathrm{~mm}$ & $-0,2 \mathrm{~mm}$ \\
Arco mandibular & $8,8^{\circ}$ & $27,7^{\circ}$ & $-4,7$ \\
Altura facial inferior & $57,7^{\circ}$ & $45,0^{\circ}$ & 3,1 \\
Relaciones dentarias & & & \\
Mx1, to APo & $8,4 \mathrm{~mm}$ & $3,5 \mathrm{~mm}$ & $2,1 \mathrm{~mm}$ \\
Mx1, to FH & $113,5^{\circ}$ & $111,0^{\circ}$ & $0,4^{\circ}$ \\
Mx6, to PTV & $6,3 \mathrm{~mm}$ & $13,1 \mathrm{~mm}$ & $-2,3 \mathrm{~mm}$ \\
Md1, to APo & $5,0 \mathrm{~mm}$ & $1,0 \mathrm{~mm}$ & $1,7 \mathrm{~mm}$ \\
Inclinación Md1 & $22,5^{\circ}$ & $22,0^{\circ}$ & 0,1 \\
Extrusión Md1 & $-7,2 \mathrm{~mm}$ & $1,2 \mathrm{~mm}$ & $-4,2 \mathrm{~mm}$ \\
Ángulo interincisivo & $117,7^{\circ}$ & $130,0^{\circ}$ & $0,3 \mathrm{~mm}$ \\
Relación molar & $-3,7 \mathrm{~mm}$ & $-3,0 \mathrm{~mm}$ & $-1,8 \mathrm{~mm}$ \\
Overjet & $3,3 \mathrm{~mm}$ & $2,5 \mathrm{~mm}$ & \\
Overbite & $-1,1 \mathrm{~mm}$ & $2,5 \mathrm{~mm}$ & \\
\hline & & & \\
& & & \\
& & & \\
& & & \\
\hline
\end{tabular}

Tabla 4. Análisis cefalométrico de tejidos blandos

\begin{tabular}{|c|c|c|c|}
\hline Variable & Resultado & Norma & Desviación clínica \\
\hline \multicolumn{4}{|l|}{ Vertical } \\
\hline Glab. - Subn. & $65,5 \mathrm{~mm}$ & $73,0 \mathrm{~mm}$ & $-0,1 \mathrm{~mm}$ \\
\hline Subn. - Submenton & $72,6 \mathrm{~mm}$ & $69,5 \mathrm{~mm}$ & $0,0 \mathrm{~mm}$ \\
\hline Sn-sMe \% of G-Sn & $111,4 \%$ & $100,0 \%$ & $1,4 \%$ \\
\hline sNasion-Subn. & $49,8 \mathrm{~mm}$ & $54,7 \mathrm{~mm}$ & $-0,0 \mathrm{~mm}$ \\
\hline Sn-sMe \% de sN-Sn & $145,8 \%$ & $132,5 \%$ & $1,3 \%$ \\
\hline Subn- StomSup & $27,4 \mathrm{~mm}$ & $20,3 \mathrm{~mm}$ & $0,1 \mathrm{~mm}$ \\
\hline StomInf- sMenton & $45,3 \mathrm{~mm}$ & $48,4 \mathrm{~mm}$ & $-0,0 \mathrm{~mm}$ \\
\hline StS-sMe \% of Sn-StS & $165,4 \%$ & $200,0 \%$ & $-3,5 \%$ \\
\hline Labio inferior-sMenton & $36,5 \mathrm{~mm}$ & $42,5 \mathrm{~mm}$ & $-0,1 \mathrm{~mm}$ \\
\hline Subn- Labio inferior & $36,1 \mathrm{~mm}$ & $27,0 \mathrm{~mm}$ & $0,1 \mathrm{~mm}$ \\
\hline Sn.Llp \% of Llp-sMe & $99,0 \%$ & $80,0 \%$ & $1,9 \%$ \\
\hline Distancia interlabial & $0,0 \mathrm{~mm}$ & $0,0 \mathrm{~mm}$ & $0,0 \mathrm{~mm}$ \\
\hline uLipEmbr - Mx1 & $0,4 \mathrm{~mm}$ & $4,9 \mathrm{~mm}$ & $-0,8 \mathrm{~mm}$ \\
\hline \multicolumn{4}{|l|}{ Sagital } \\
\hline Subn. Perp- Soft-A & $-2,5 \mathrm{~mm}$ & $-2,0 \mathrm{~mm}$ & $-0,2 \mathrm{~mm}$ \\
\hline Subn. Perp- uLip & $-1,1 \mathrm{~mm}$ & $3,0 \mathrm{~mm}$ & $-1,4 \mathrm{~mm}$ \\
\hline Subn. Perp- 1Lip & $-1,1 \mathrm{~mm}$ & $1,0 \mathrm{~mm}$ & $-0,7 \mathrm{~mm}$ \\
\hline Subn. Perp- Soft-B & $-11,6 \mathrm{~mm}$ & $-5,0 \mathrm{~mm}$ & $-2,2 \mathrm{~mm}$ \\
\hline Subn. Perp- Mentón & $-17,9 \mathrm{~mm}$ & $-2,0 \mathrm{~mm}$ & $-5,3 \mathrm{~mm}$ \\
\hline \multicolumn{4}{|l|}{ Combinación } \\
\hline Subn. - sGnation & $72,8 \mathrm{~mm}$ & $69,7 \mathrm{~mm}$ & $0,0 \mathrm{~mm}$ \\
\hline sGnathion-Cervical & $45,6 \mathrm{~mm}$ & $55,2 \mathrm{~mm}$ & $-0,1 \mathrm{~mm}$ \\
\hline sGn-Cerv \% of Sn-sGn & $62,6 \%$ & $80,0 \%$ & $-1,7 \%$ \\
\hline Subn. - Nariz & $8,7 \mathrm{~mm}$ & $17,5 \mathrm{~mm}$ & $-0,1 \mathrm{~mm}$ \\
\hline Ángulo nasolabial & $140,7^{\circ}$ & $115,2^{\circ}$ & 4,3 \\
\hline
\end{tabular}


volver la línea media superior e inferior se coordinaron ambas arcadas y se utilizó ligaduras metálicas conjugadas en ocho. Los arcos superiores e inferiores cambiaron a Nitinol 0,016", Nitinol 0,016 x 0,016, Acero (SS) 0,016 x 0,016. Se retiró la barra lingual y se cambió la barra palatina por un aparato de expansión tipo Hass modificado, se activó hasta lograr la expansión necesaria del maxilar. Una vez retirada la barra, se utilizó fuerza elástica intraoral con indicación de elásticos intraoral
1/4”. En las Figuras 5 y 6 se puede observar secuencia de proceso de tratamiento ortodóntico.

Una vez lograda la alienación de las arcadas superior e inferior, junto con obtener una oclusión estable, se retiraron los aparatos fijos superiores e inferiores y se instalaron aparatos removibles tipo Hawley (Figura 7) para la contención en ambas arcadas, que fueron controlados de forma mensual. La fase ortodóntica tuvo una duración de 4 años.
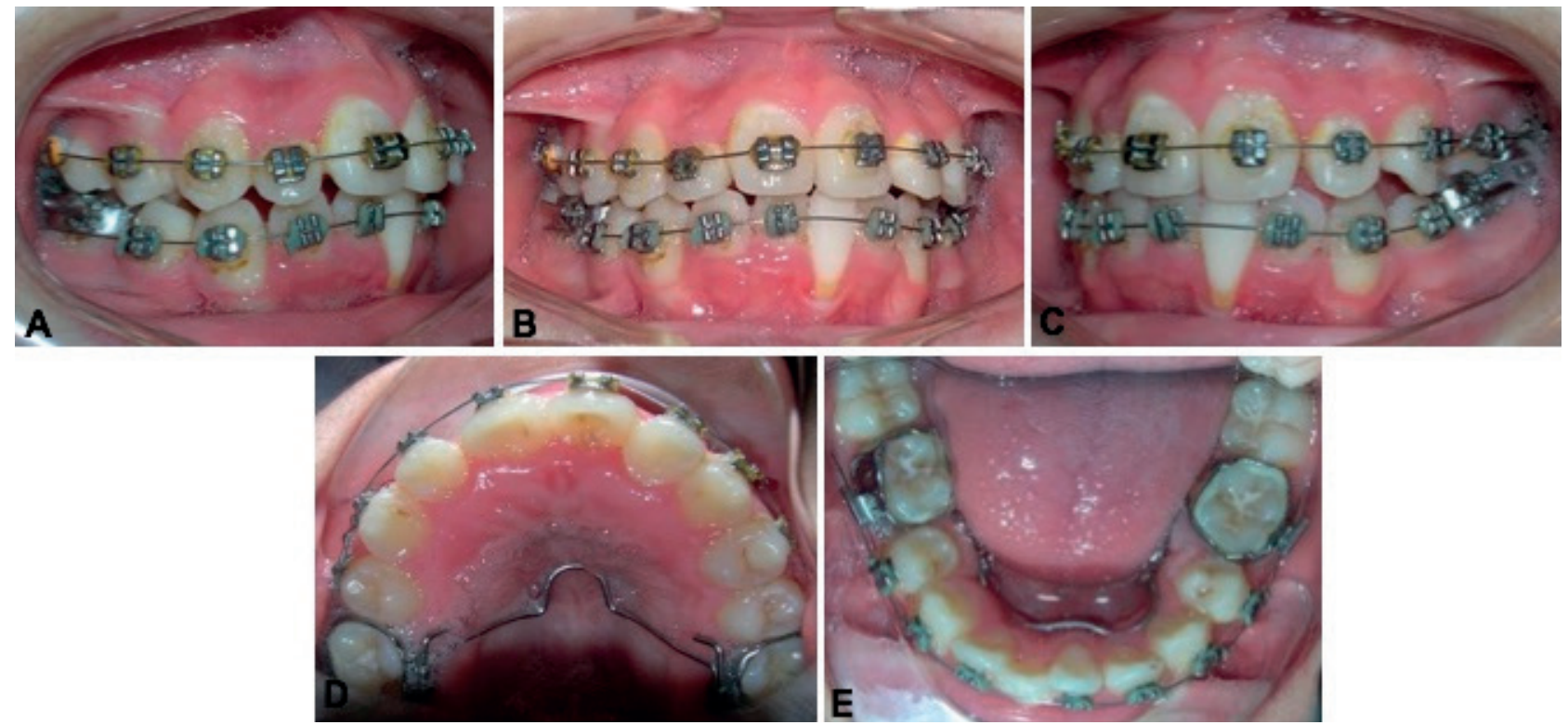

Figura 5. A. Oclusión lado derecho. B. Oclusión de frente. C. Oclusión lado izquierdo. D. Arcada superior, se observa barra de expansión maxilar y E. Arco inferior con barra lingual
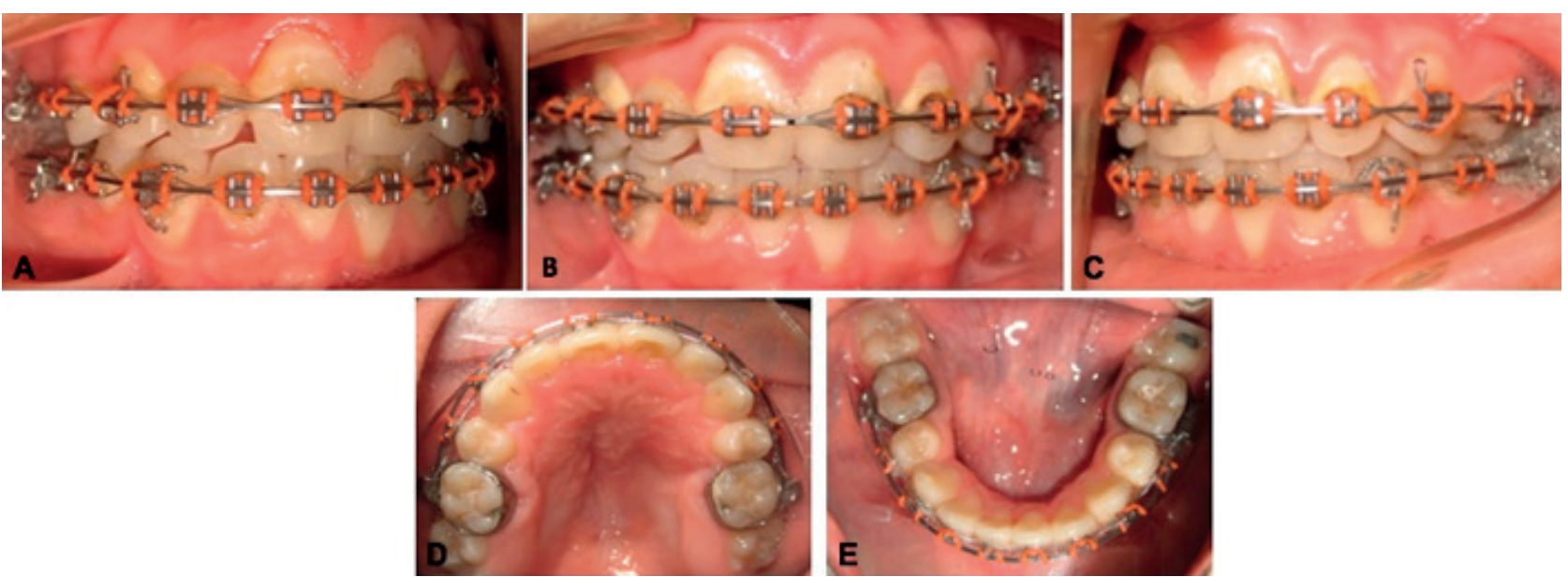

Figura 6. A. Oclusión lado derecho. B. Oclusión de frente. C. Oclusión lado izquierdo. D. Arcada superior. E. Arcada inferior
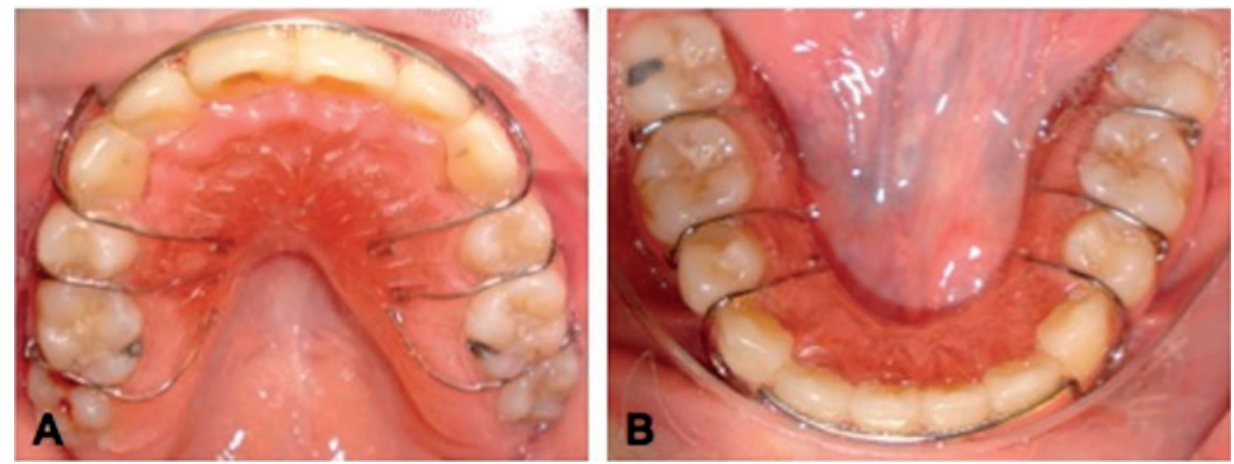

Figura 7. A. y B. Arcos superior e inferior con contenciones removibles, post tratamiento ortodóntico 
Durante uno de sus controles post ortodoncia se observó recidiva a los 2 años de control, en el arco inferior, pieza 4.3 en malposición, y presencia de diastemas en sector antero inferior. Se cementan multibrackets en arco inferior con arco de Nitinol 0.014", Nitinol $0.016 "$, Nitinol 0,018”, SS 0,016" x $0.016 "$, SS $0.017^{*}$ x $0.025^{\circ}$, uso de Alastic en una primera etapa y luego uso de ligaduras metálicas en ocho. Una vez controlada la recidiva, se coloca nueva contención inferior, que se controla mensualmente, insistiendo en el uso. En la Figura 8 se observa finalizado el tratamiento ortodóntico.
Dentro de las dificultades del tratamiento estuvo la falta de motivación del paciente para mantener una buena higiene antes y después del tratamiento ortodóntico, ya que durante el tiempo de intervención se insistió, se educó y se repitió constantemente, se realizaron instrucciones de higiene, destartrajes supragingivales y pulidos coronarios, pero, aun así, no se logró una buena cooperación del paciente.

Durante el seguimiento, 3 años después del retiro de aparatos fijos de ortodoncia, se observó en el maxilar
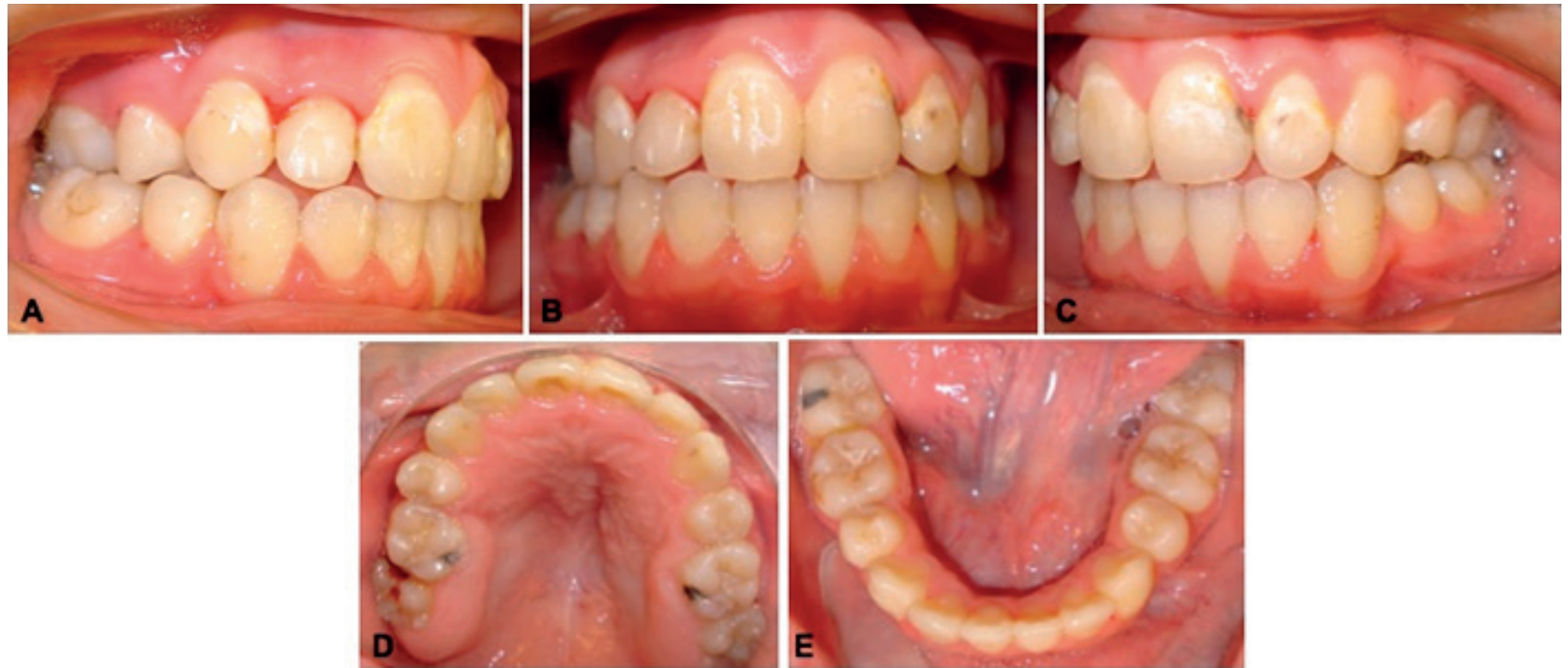

Figura 8. A, B y C. Oclusión en máxima intercuspidación. D y E. Arcos superior e inferior finalizado tratamiento ortodóntico
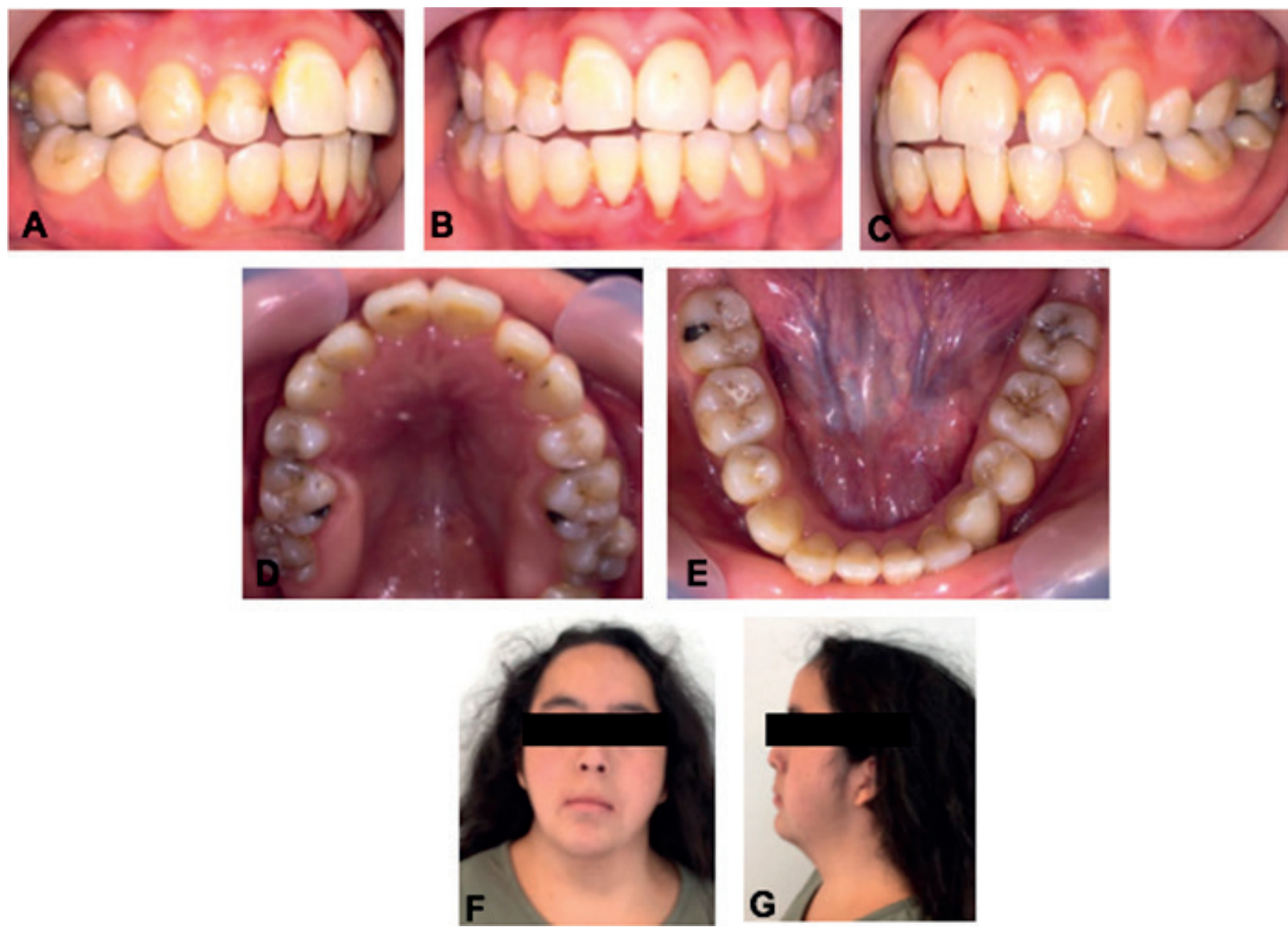

Figura 9. Seguimiento de tratamiento a los 10 años. A, B y C. Oclusión lado derecho, de frente, lado izquierdo. D y E. Arcadas superior e inferior. $\mathbf{F}$ y G. Fotografía facial frontal y de perfil 
la aparición de diastema entre piezas 2.1-2.2, palatinización de pieza 1.2, debido a que dejó de usar la placa de contención superior. Además, se observó inflamación gingival en grupo antero inferior.

\section{Discusión}

En pacientes con síndrome de Turner se pueden distinguir alteraciones de la oclusión relacionadas con este conjunto de expresiones clínicas. Según estudios, son frecuentes las posiciones retrognáticas del maxilar y la mandíbula ${ }^{4}$. Para realizar un plan de tratamiento es necesario considerar en estos pacientes, la presencia de tratamiento hormonal y también la necesidad de profilaxis antibiótica en casos de que existan problemas cardíacos, cuando sea necesario realizar alguna cirugía ${ }^{8}$.

En casos de maloclusión acompañadas de alguna alteración muscular facial o incluso la presencia de malos hábitos, tales como, la respiración bucal, succión digital o una deglución atípica, el tratamiento ortodóntico no es suficiente para asegurar el éxito de un tratamiento que mantenga la estabilidad. Es necesario, además considerar combinarlo con terapia miofuncional. Debido a que la musculatura es un factor importante para la estabilidad de la oclusión, su disfunción puede influir en el desarrollo craneofacial ${ }^{9,10}$. La maloclusión, dependiendo del caso, puede ser corregida con elementos interceptivos, ortodoncia fija y en algunos casos se ha requerido cirugía ortognática ${ }^{10,11}$.

En el presente caso clínico, la derivación fue realizada para tratamiento y se consideró como prioridad la hipofunción muscular que presenta la paciente, pero la condición oclusal también requería resolución. Es así como fue necesario realizar un tratamiento interdisciplinario, que permitiera rehabilitar y educar una musculatura hipotónica, como consecuencia de un síndrome de Turner, eliminando así los factores que pueden perpetuar la maloclusión, y junto con eso, restablecer una oclusión funcional mediante tratamiento ortodóntico.

La recidiva que se obtuvo en la paciente fue consecuencia de la falta de uso de las contenciones removibles, y no corresponde a una recaída en el funcionamiento de la musculatura. Si bien, las intervenciones musculares se realizaron en la primera fase, se lograron resultados positivos, que se mantuvieron durante todo el resto del tratamiento.

A pesar del movimiento de algunas piezas dentales, luego del seguimiento que se realizó, se ha mantenido una oclusión estable y un equilibrio muscular que permite la función de las estructuras craneofaciales, logrando de esta manera, el objetivo inicial del tratamiento complementario.

\section{Referencias bibliográficas}

1. Saenger P, Albertsson Wikland K, Conway GS, Daveport M, Graveholt CH, Hintz R, et al. Recommendations for the Diagnosis and Management of Turner Syndrome. J Clin Endocrinol Metab. 2001;86:3061-3069. DOI: 10.1210/jcem.86.7.7683

2. Ross JL, Kushner H, Roeltgen DP. Developmental Changes in Motor Function in Girls with Turner Syndrome. Pediatr Neurol. 1996;15:317-322.

3. Dumancic J, Kaic Z, Lapter Varga M, Lauc T, Dumic M, Anic Milosevic S, et al. Characteristics of the craniofacial complex in Turner Syndrome. Arch Oral Biol. 2010;55:81-88. DOI: 10.1016/j.archoralbio.2009.10.008

4. Svanberg C, Norevall Ll, Ekman B, Wahlberg J, Bågesund M. Cephalometric analysis of adults with Turner Syndrome. Swed Dent J. 2016;40:33- 41.

5. Haverkamp F, Keuker T, Woelfle J, Kaiser G, Zerres K, Rietz $\mathrm{C}$, et al. Familiar factors and hearing impairment modulate the neuromotor phenotype in Turner Syndrome. Eur J Pediatr. 2003;162:30- 35. DOI: 10.1007/ s00431-002-1087-5

6. Nijhuis-van der Sanden RWG, Smits-Engelsman BCM, Eling PATM. Motor performance in girls with Turner Syndrome. Dev Med Child Neurol. 2000;42:685-690.

7. Salbenblatt JA, Meyers DC, Bender BG, Linden MG, Robinson A. Gross and Fine Motor Development in 45,X and 47,XXX Girls. Pediatrics. 1989. Oct; $84(4): 678-682$.

8. Russell KA. Orthodontic treatment for patients with Turner syndrome. Am J Orthod Dentofacial Orthop. 2001 Sep;120(3):314- 322. DOI: 10.1067/mod.2001.115719

9. Saccomanno S, Antonini G, D'Alatri L, D'Angelantonio M, Florita A, Deli A. Patients treated with ortohontic-myofuncional therapeutic protocol. Eur J Pediatr Dent. 2012;13:241-243.

10. Sugawara $Y$, Ishihara Y, Takano-Yamamoto T, Yamashiro T, Kamioka H. Orthodontic treatment of a patient with unilateral orofacial muscle dysfunction: The efficacy of myofuncional therapy on the treatment outcome. Am J Orthod Dentofacial Orthop. 2016;150:167-180. DOI: 10.1016/j.ajodo.2015.08.021

11. Aristizábal JF, Smit RM. Two-Phase Orthodontic Treatment in a Patient with Turner Syndrome: An Unusual Case of Deep Bite. Cleft Palate Craniofac J. 2015;52(3):e56-e64. DOI: 10.1597/13-157 